\title{
Path Diversity in Packet Switched Networks: Performance Analysis and Rate Allocation
}

\author{
Shervan Fashandi, Shahab Oveis Gharan and Amir K. Khandani \\ ECE Dept., University of Waterloo, Waterloo, ON, Canada, N2L3G1 \\ email: $\{$ sfashand,shahab,khandani\}@ cst.uwaterloo.ca
}

\begin{abstract}
Path diversity works by setting up multiple parallel connections between the end points using the topological path redundancy of the network. In this paper, Forward Error Correction (FEC) is applied across multiple independent paths to enhance the end-to-end reliability. Internet paths are modeled as erasure Gilbert-Elliot channels [1], [2]. First, it is shown that over any erasure channel, Maximum Distance Separable (MDS) codes achieve the minimum probability of irrecoverable loss among all block codes of the same size. Then, we prove the probability of irrecoverable loss decays exponentially for the asymptotically large number of paths. Moreover, it is shown that in the optimal rate allocation, each path is assigned a positive rate iff its quality is above a certain threshold. The quality of a path is defined as the percentage of the time it spends in the bad state. Finally, using dynamic programming, a heuristic suboptimal algorithm with polynomial runtime is proposed for rate allocation over the available paths. This algorithm converges to the asymptotically optimal rate allocation when the number of paths is large. The simulation results show that the proposed algorithm approximates the optimal rate allocation very closely, and provides significant performance improvement compared to the alternative schemes of rate allocation. ${ }^{1}$
\end{abstract}

\section{INTRODUCTION}

In recent years, path diversity over the Internet has received significant attention. It has been shown that path diversity has the ability to simultaneously improve the end-to-end rate and reliability [2], [3]. In a dense network like the Internet, it is usually possible to find multiple disjoint paths between any pair of nodes [4]. In this paper, Forward Error Correction (FEC) is applied across multiple disjoint paths. Knowing that packet loss and delay patterns are independent over such paths, we expect path diversity to enhance the performance of FEC.

Several centralized and distributed algorithms are proposed to find multiple independent paths over a large connected graph [5]. Although the distributed algorithms do not require the end nodes to know the entire topology of the network, they impose some modifications on the intermediate nodes. Indeed, modification of the routing protocol and the signaling between the nodes is extremely costly over the traditional IP networks. To avoid such an expense, overlay networks are introduced [6]. The basic idea of overlay networks is to equip a few number of nodes (smart nodes) with the desired new functionalities while the rest remain unchanged. The smart nodes form a

\footnotetext{
${ }^{1}$ Financial support provided by Nortel and the corresponding matching funds by the Natural Sciences and Engineering Research Council of Canada (NSERC), and Ontario Centres of Excellence (OCE) are gratefully acknowledged.
}

virtual network connected through logical links on top of the actual network. Thus, the overlay networks can be used to set up disjoint paths between the end nodes. Reference [4] has experimentally studied the number of available disjoint paths in the Internet using overlay networks.

Recently, path diversity is utilized in many applications [2], [7], [8]. Reference [8] combines multiple description coding and path diversity to improve quality of service (QoS) in video streaming. Moreover, references [3] and [2] study the problem of rate allocation over multiple paths. Assuming each path follows the leaky bucket model, reference [3] shows that a water-filling based scheme provides the minimum endto-end delay. On the other hand, reference [2] considers a scenario of multiple senders and a single receiver, assuming all the senders share the same source of data. The connection between each sender and the receiver is assumed to follow the Gilbert-Elliot model. They propose a receiver-driven protocol for packet partitioning and rate allocation. The rate allocation algorithm minimizes the probability of irrecoverable loss in the FEC scheme [2]. They apply a brute-force search algorithm to solve the rate allocation problem over two paths only. However, it should be noted that the scenario of [2] can be simplified, without any loss of generality, into the case of multiple independent paths between a pair of end nodes as the senders share the same data.

Maximum Distance Separable (MDS) codes has been shown to be optimum in the sense that they achieve the maximum possible minimum distance $\left(d_{\text {min }}\right)$ among block codes of the same size [9]. Indeed, any $[n, k]$ MDS code with block length $n$ and $k$ symbols of information can be successfully recovered from any subset of its entries of length $k$ or more. This property makes MDS codes favorable FEC schemes over the erasure channels like the Internet [10], [11].

In this work, we utilize path diversity to improve the performance of FEC. Probability of irrecoverable loss $\left(P_{E}\right)$ is defined as the measure of FEC performance. First, it is shown that MDS block codes have the minimum probability of error over any erasure channel. Our analysis shows an exponential decay of $P_{E}$ with respect to the number of paths, $L$. Moreover, the optimal rate allocation problem is solved in the asymptotic case where the number of paths becomes very large. It is seen that in the optimal rate allocation, each path is assigned a positive rate iff its quality is above a certain threshold. Furthermore, using dynamic programming, a heuristic suboptimal algorithm is proposed for rate allocation over the available 
paths. Unlike the brute-force search, this algorithm has a polynomial complexity in terms of $L$. It is shown that the result of this algorithm converges to the asymptotically optimal solution for large number of paths.

The rest of this paper is organized as follows. Section II describes the system model. Performance of FEC in the two cases of multiple identical paths, and non-identical paths are analyzed in section III. Section IV studies the rate allocation problem, and proposes a suboptimal rate allocation algorithm. Finally, section V concludes the paper.

\section{System Modeling ANd Formulation}

\section{A. Internet Channel Model}

From an end to end protocol's perspective, performance of the lower layers in the protocol stack can be modeled as a random channel called Internet channel. Since each packet usually includes an internal error detection coding (for instance a CRC), the Internet channel is satisfactorily modeled as an erasure channel. Although there is no well defined capacity limit for the Internet channel, a maximum TCP-friendly rate is introduced in [12].

In this work, the model assumed for the Internet channel is a two-state Markov model called Gilbert-Elliot cell. The channel spends an exponentially distributed random amount of time with the mean $\frac{1}{\mu_{g}}$ in the Good state. Then, it alternates to the Bad state and stays in that state for another random duration exponentially distributed with the mean $\frac{1}{\mu_{b}}$. If a packet is transmitted from the source at anytime during the good state, it will be received correctly. Otherwise, if it is transmitted during the bad state, it will eventually be lost before reaching the destination. Therefore, the average probability of error is equal to the steady state probability of being in the bad state $\pi_{b}=\frac{\mu_{g}}{\mu_{q}+\mu_{b}}$. To have a reasonably low probability of error, $\mu_{g}$ must be much smaller than $\mu_{b}$.

\section{B. Typical FEC Model}

A concatenated coding is used for packet transmission. The coding inside each packet can be a simple CRC which enables the receiver to detect an error inside each packet. Then, the receiver can consider the Internet channel as an erasure channel. Other than the coding inside each packet, a Forward Error Correction (FEC) scheme is applied between packets. Every $K$ packets are encoded to a Block of $N$ packets where $N>K$ to create some redundancy. The ratio of $\alpha=\frac{N-K}{N}$ defines the FEC overhead. A Maximum Distance Separable (MDS) $(N, K)$ code, such as Reed-Solomon code, can reconstruct the original $K$ data packets on the receiver side if $K$ or more of the $N$ packets are received correctly [9]. According to the following proposition, a MDS code is the optimum block code we can design over any erasure channel.

Proposition I. An erasure channel is defined as the one which maps every input symbol to either itself or an erasure symbol $\xi$. Consider an arbitrary erasure channel (memoryless or with memory) with the input vector $\mathrm{x} \in \mathcal{X}^{N},|\mathcal{X}|$, the output vector $\mathbf{y} \in(\mathcal{X} \cup\{\xi\})^{N}$, and the transition probability $p(\mathbf{y} \mid \mathbf{x})$ satisfying:
1) $p\left(y_{j} \notin\left\{x_{j}, \xi\right\} \mid x_{j}\right)=0$.

2) $p(\mathbf{y} \mid \mathbf{x})$ is independent of the input vector $\mathbf{x}$ if $\forall j y_{j} \in$ $\left\{x_{j}, \xi\right\}$.

A block code $(N, K)$ with equiprobable codewords over this channel has the minimum probability of error using the optimum (maximum likelihood) decoder among all block codes of the same size iff that code is Maximum Distance Separable (MDS). The proof can be found in [13].

\section{Rate Allocation Problem}

Let us assume all packets have the equal length of $r$ bits. Then, all rates can be expressed in pkt/sec instead of bps. From source to the destination, there exist $L$ independent paths with maximum rates of $W_{i}$ each. $W_{i}$ can be considered as the maximum TCP-friendly bandwidth introduced in reference [12] or any other upperbound imposed by the physical characteristics of the $i$ 'th path. For a specific application and FEC scheme, we require the rate $S_{r e q}$ from source to the destination. Obviously, we should have $S_{r e q} \leq \Sigma W_{i}$ to have a feasible solution. The total time to send a block of $N$ packets is $T=\frac{N}{S_{r e a}}$. The block length is typically much larger than the number of paths $(N \gg L)$. According to the FEC model, we can send $N_{i}$ packets through the path $i$ as long as $\sum N_{i}=N$ and $\frac{N_{i}}{T} \leq W_{i}$. Then, the rate assigned to path $i$ can be expressed as $S_{i}=\frac{N_{i}}{T}=\frac{N_{i}}{N} S_{r e q}$. The objective of the rate allocation problem is to find the optimal rate allocation vector or the vector $\mathbf{N}=\left(N_{1}, \cdots, N_{L}\right)$ which minimizes the probability of irrecoverable loss $\left(P_{E}\right)$. In this work, an irrecoverable loss is defined as the event where more than $K$ packets are lost in a block of $N$ packets.

\section{Discrete to Continuous Approximation}

The continuous random variable $B_{i}$ is defined as the duration of time that the path $i$ spends in the bad state in a block duration, $T$. We denote the values of $B_{i}$ with parameter $t$ to emphasize that they are expressed in the time unit. The probability distribution of $B_{i}, f_{B_{i}}(t)$ is computed in [13].

To detect an irrecoverable loss, we are interested in the probability of $k_{i}$ packets being lost out of the $N_{i}$ packets transmitted through the path $i$. Let us denote the number of erroneous or lost packets with the random variable $E_{i}$. Any two subsequent packets in a block are $\frac{1}{S_{i}}$ seconds apart in time, where $S_{i}$ is the transmission rate over the path $i$. Assuming there can be at most one bad burst over a block, we observe that the probability $\mathbb{P}\left\{E_{i} \geq k_{i}\right\}$ can be approximated with the continuous counterpart $\mathbb{P}\left\{B_{i} \geq \frac{k_{i}}{S_{i}}\right\}$. This approximation is reasonable when the inter-packet interval is much shorter than the typical bad burst, $\frac{1}{S} \ll \mathbb{E}\{B\}$ (Throughout this paper, $\mathbb{E}\{\}$ stands for the expected value operator). The continuous approximation simplifies the mathematical analysis of section III.

\section{Performance Analysis of FEC on Multiple PATHS}

Assume that a rate allocation algorithm assigns $N_{i}$ packets to the path $i$. When the $N_{i}$ packets of the FEC block are 
sent over path $i$, the loss count can be approximated as $\frac{B_{i}}{T} N_{i}$. Hence, The total ratio of lost packets is equal to

$$
\sum_{i=1}^{L} \frac{B_{i} N_{i}}{T N}=\sum_{i=1}^{L} \frac{B_{i} \rho_{i}}{T}
$$

where $\rho_{i}=\frac{S_{i}}{S_{\text {req }}}, 0 \leq \rho_{i} \leq 1$, denotes the portion of bandwidth assigned to the path $i . x_{i}=\frac{B_{i}}{T}$ is defined as the portion of time the path $i$ has been in the bad state $\left(0 \leq x_{i} \leq 1\right)$. Hence, the probability of irrecoverable loss is equal to

$$
P_{E}=\mathbb{P}\left\{\sum_{i=1}^{L} \rho_{i} x_{i}>\alpha\right\}
$$

where $\alpha=\frac{N-K}{N}$. In order to find the optimum rate allocation, $P_{E}$ has to be minimized with respect to the allocation vector $\left(\rho_{i}\right.$ 's), subject to the following constraints

$$
0 \leq \rho_{i} \leq \min \left\{1, \frac{W_{i}}{S_{r e q}}\right\}, \quad \sum_{i=1}^{L} \rho_{i}=1
$$

where $W_{i}$ is the TCP-friendly bandwidth defined in subsection II-C. The distribution of $x_{i}$ 's are given and proportional to that of $B_{i}$ 's.

\section{A. Identical Paths}

When the paths are identical, due to the symmetry of the problem, the uniform rate allocation $\left(\rho_{i}=\frac{1}{L}\right)$ is obviously the optimum solution. Then, $P_{E}$ can be simplified as $P_{E}=$ $\mathbb{P}\left\{\frac{1}{L} \sum_{i=1}^{L} x_{i}>\alpha\right\}$. Let us define $Q(x)$ as the probability distribution function of $x$. There is a well-known upperbound for the above probability in large deviation theory [14]

$$
u(\alpha)= \begin{cases}0 & P_{E} \leq e^{-u(\alpha) L} \\ \lambda \alpha-\log \left(\mathbb{E}\left\{e^{\lambda x}\right\}\right) & \text { for } \alpha \leq \mathbb{E}\{x\}\end{cases}
$$

where $\lambda$ is the solution of the following non-linear equation, which will be shown to be unique by Lemma I.

$$
\alpha=\frac{\mathbb{E}\left\{x e^{\lambda x}\right\}}{\mathbb{E}\left\{e^{\lambda x}\right\}} .
$$

Since $\lambda$ is unique, we can define $l(\alpha)=\lambda$. Even though an upperbound, inequality (3) is shown to be exponentially tight for large values of $L$ [14]. More precisely, we have $P_{E} \doteq$ $e^{-u(\alpha) L}$, where the notation $\doteq$ means $\lim _{L->\infty}-\frac{\log P_{E}}{L}=$ $u(\alpha)$. Now, we state two useful lemmas whose proofs can be found in [13].

Lemma I. $u(\alpha)$ and $l(\alpha)$ have the following properties:

1) $\frac{\partial}{\partial \alpha} l(\alpha)>0$

2) $l(\alpha=0)=-\infty, l(\alpha=\mathbb{E}\{x\})=0, l(\alpha=1)=+\infty$

3) $\frac{\partial}{\partial \alpha} u(\alpha)=l(\alpha)>0$ for $\alpha>\{x\}$

Lemma II. Defining $y=\frac{1}{L} \sum_{i=1}^{L} x_{i}$, where $x_{i}$ 's are i.i.d. random variables as already defined, the probability density function of $y$ has the property of $f_{y}(\alpha) \doteq e^{-u(\alpha) L}$, for all $\alpha>\mathbb{E}\{x\}$.

Fig. 1 compares the theoretical and simulation results for a connection with the aggregated bandwidth of $S_{r e q}=$

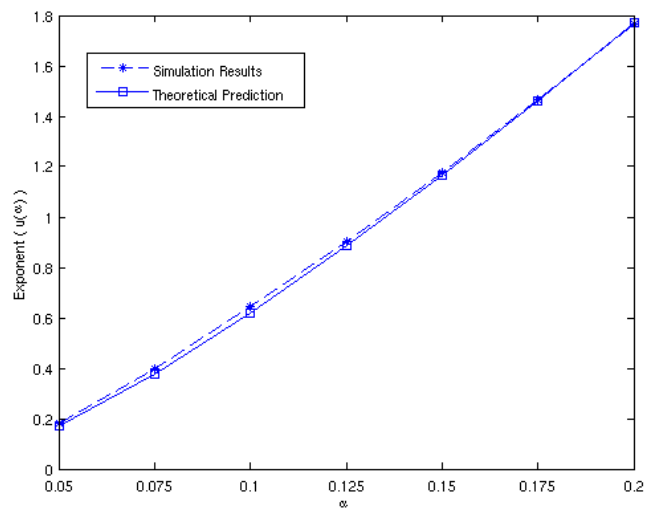

Fig. 1. The decaying exponent (slope) of $P_{E}$ versus $L$ for different values of $K$ : experimental versus theoretical values.

$1000 \mathrm{pkt} / \mathrm{s}$, average bad and good burst lengths of $\frac{1}{\mu_{b}}=$ $15.0 \mathrm{~ms}$ and $\frac{1}{\mu_{g}}=1000 \mathrm{~ms}$, and the block length of $N=200$ packets. The number of the information packets varies from $K=160$ to $K=190$. $P_{E}$ is plotted versus $L$ in semilogarithmic scale in [13] for every fixed value of $K$. We observe that as $L$ increases, $P_{E}$ decays exponentially which is expectable from equation (3). Also, Fig. 1 compares the slope (exponent) of such plots with $u(\alpha)$. Fig. 1 shows a good agreement between the theory and the simulation results, and also verifies the fact that the stronger the FEC code is (smaller $K$ ), the more gain we achieve from path diversity (larger exponent).

\section{B. Non-Identical Paths}

Now, let us assume there are $J$ types of paths between the source and the destination, consisting of $L_{j}$ identical paths from type $j\left(\sum_{j=1}^{J} L_{j}=L\right)$. Without loss of generality, we can assume that the paths are ordered according to their associated type, i.e. the paths from $1+\sum_{k=1}^{j-1} L_{k}$ to $\sum_{k=1}^{j} L_{k}$ are of type $j$. We denote $\gamma_{j}=\frac{L_{j}}{L}$. According to the i.i.d. assumption, it is obvious that $\rho_{i}$ has to be the same for all paths from the same type. $\eta_{j}$ and $y_{j}$ are defined as

$$
\begin{aligned}
\eta_{j} & =\sum_{\sum_{k=1}^{j-1} L_{k}<i \leq \sum_{k=1}^{j} L_{k}} \rho_{i} \\
y_{j} & =\frac{\eta_{j}}{L \gamma_{j}} \sum_{\sum_{k=1}^{j-1}} \sum_{L_{k}<i \leq \sum_{k=1}^{j} L_{k}} x_{i} .
\end{aligned}
$$

Following Lemma II, we observe that $f_{y_{j}}\left(\beta_{j}\right) \doteq e^{-\gamma_{j} u_{j}\left(\frac{\beta_{j}}{\eta_{j}}\right) L}$. We define the sets $\mathcal{S}_{I}, \mathcal{S}_{O}$ and $\mathcal{S}_{T}$ as

$$
\begin{aligned}
& \mathcal{S}_{I}=\left\{\left(\beta_{1}, \beta_{2}, \cdots, \beta_{J}\right) \mid 0 \leq \beta_{j} \leq 1, \sum_{j=1}^{J} \beta_{j}>\alpha\right\} \\
& \mathcal{S}_{O}=\left\{\left(\beta_{1}, \beta_{2}, \cdots, \beta_{J}\right) \mid 0 \leq \beta_{j} \leq 1, \sum_{j=1}^{J} \beta_{j}=\alpha\right\} \\
& \mathcal{S}_{T}=\left\{\left(\beta_{1}, \beta_{2}, \cdots, \beta_{J}\right) \mid \eta_{j} \mathbb{E}\left\{x_{j}\right\} \leq \beta_{j}, \sum_{j=1}^{J} \beta_{j}=\alpha\right\},
\end{aligned}
$$


respectively. Hence, $P_{E}$ can be written as

$$
\begin{aligned}
P_{E} & =\mathbb{P}\left\{\sum_{j=1}^{J} y_{j}>\alpha\right\}=\oint_{\mathcal{S}_{I}} \prod_{j=1}^{J} f_{y_{j}}\left(\beta_{j}\right) d \beta_{j} \\
& \doteq \oint_{\mathcal{S}_{I}} e^{-L \sum_{j=1}^{J} \gamma_{j} u_{j}\left(\frac{\beta_{j}}{\eta_{j}}\right)} d \beta_{j} \\
& \stackrel{(a)}{\doteq} e^{-L} \min _{\beta \in \mathcal{S}_{I} \cup \mathcal{S}_{O}} \sum_{j=1}^{J} \gamma_{j} u_{j}\left(\frac{\beta_{j}}{\eta_{j}}\right) \\
& \stackrel{(b)}{\doteq} e^{-L \min _{\beta \in \mathcal{S}_{O}} \sum_{j=1}^{J} \gamma_{j} u_{j}\left(\frac{\beta_{j}}{\eta_{j}}\right)} \\
& \stackrel{(c)}{\doteq} e^{-L \min _{\beta \in \mathcal{S}_{T}} \sum_{j=1}^{J} \gamma_{j} u_{j}\left(\frac{\beta_{j}}{\eta_{j}}\right)}
\end{aligned}
$$

where $(a)$ follows from reference [13], $(b)$ is resulted from the fact $u_{j}(\alpha)$ is a strictly increasing function of $\alpha$, for $\alpha>\mathbb{E}\left\{x_{j}\right\}$, and $(c)$ follows from the property that $u_{j}(\alpha)=$ $0, \forall \alpha \leq \mathbb{E}\left\{x_{j}\right\}$.

Equation (6) is valid for any fixed value of the vector $\eta$. To achieve the most rapid decay of $P_{E}$, the exponent must be maximized over $\eta$.

$$
\lim _{L->\infty}-\frac{\log P_{E}}{L}=\max _{0 \leq \eta_{j} \leq 1, \sum_{j=1}^{J}} \eta_{j}=1 \min _{\beta \in \mathcal{S}_{T}} \sum_{j=1}^{J} \gamma_{j} u_{j}\left(\frac{\beta_{j}}{\eta_{j}}\right)
$$

Lemma III solves the maximization problem of (7) and identifies the asymptotically optimum rate allocation (for large number of paths).

Lemma III. The optimization problem of equation (7) has a unique solution, $\eta^{\star}$, with the elements

$$
\eta_{j}^{\star}= \begin{cases}\frac{\gamma_{j} l_{j}(\alpha)}{\sum_{i=1, \alpha \leq \mathbb{E}\left\{x_{i}\right\}}^{J} \gamma_{i} l_{i}(\alpha)} & \text { if } \alpha \leq \mathbb{E}\left\{x_{j}\right\} \\ \text { otherwise }\end{cases}
$$

if there is at least one $1 \leq j \leq J$ for which $\alpha>\mathbb{E}\left\{x_{j}\right\}$. The maximum value of the objective function is $\sum_{j=1}^{J} \gamma_{j} u_{j}(\alpha)$. The proof of Lemma III can be found in [13].

\section{Suboptimal Rate Allocation}

In order to compute the complexity of the rate allocation problem, we go back to the original discrete formulation in subsection II-C. According to the model of subsection III-B, we assume the available paths are from $J$ types, $L_{j}$ paths from type $j$, such that $\sum_{j=1}^{J} L_{j}=L$. Obviously, all the paths from the same type should take equal rate. Therefore, the rate allocation problem is turned into finding the vector $\mathbf{N}=\left(N_{1}, \ldots, N_{J}\right)$ such that $\sum_{j=1}^{J} N_{j}=N$, and $0 \leq N_{j} \leq$ $L_{j} W_{j} T$ for all $j . N_{j}$ denotes the number of packets assigned to the paths of type $j$ all together. It is easy to see that the number of candidate vectors, mathbf $N$, is exponential in terms of $J$ or $N$ [13].

Let us define $P_{e}^{\mathbf{N}}(k, j)$ as the probability of having more than $k$ errors over the paths of types 1 to $j$ for a specific allocation vector $\mathbf{N}$. We also define $Q_{j}(n, k)$ as the probability of having exactly $k$ errors out of the $n$ packets sent over the paths of type $j . Q_{j}(n, k)$ can be computed and stored for all path types and values of $n$ and $k$ with polynomial complexity as explained in [13]. Then, using dynamic programming, $P_{e}^{\mathbf{N}}(k, j)$ can be computed with the complexity of $O\left(K^{2} J\right)$ [13]. Therefore, the problem of rate allocation is NP in the sense that $P_{E}$ can be computed in polynomial time for any candidate vector $\mathbf{N}=\left(N_{1}, \ldots, N_{J}\right)$.

Now, we propose a suboptimal polynomial time algorithm to find the best path allocation vector, $\mathbf{N}^{o p t}$. Let us define $P_{e}^{o p t}(n, k, j)$ as the probability of having more than $k$ errors for a block of length $n$ over the paths of types 1 to $j$ minimized over all possible rate allocations $\left(\mathbf{N}=\mathbf{N}^{o p t}\right)$. First, we find a lowerbound $\hat{P}_{e}(n, k, j)$ for $P_{e}^{o p t}(n, k, j)$ from the following recursive formula

$$
\begin{aligned}
& \hat{P}_{e}(n, k, j)= \begin{cases}\min _{0 \leq n_{j} \leq \min \left\{n,\left\lfloor L_{j} W_{j} T\right\rfloor\right\}} \sum_{i=0}^{n_{j}} Q_{j}\left(n_{j}, i\right) . \\
\hat{P}_{e}\left(n-n_{j}, k-i, j-1\right) & \text { if } k \geq 0 \\
1 & \text { if } k<0\end{cases} \\
& \hat{P}_{e}(n, k, 1)=\sum_{i=k+1}^{n} Q_{1}(n, i) .
\end{aligned}
$$

Using dynamic programming, it is easy to show that the above formula computes $\hat{P}_{e}(N, K, J)$ with the complexity of $O\left(N^{2} K^{2} J\right)$, and $\hat{P}_{e}(n, k, j)$ is in fact a lowerbound for $P_{e}^{o p t}(n, k, j)$ [13]. The following algorithm recursively finds a suboptimum allocation vector $\hat{\mathbf{N}}$ based on this lowerbound.

(1): Initialize $j \leftarrow J, n \leftarrow N, k \leftarrow K$.

(2): Set

$$
\begin{aligned}
\hat{N}_{j}= & \underset{\substack{0 \leq n_{j} \leq \min \left\{n,\left\lfloor L_{j} W_{j} T\right\rfloor\right\} \\
\hat{P}_{e}\left(n-n_{j}, k-i, j-1\right)}}{\operatorname{argmin}} \sum_{i=0}^{n_{j}} Q_{j}\left(n_{j}, i\right) . \\
K_{j}= & \underset{0 \leq i \leq \hat{N}_{j}}{\operatorname{argmax}} \hat{P}_{e}\left(n-\hat{N}_{j}, k-i, j-1\right) Q_{j}\left(\hat{N}_{j}, i\right)
\end{aligned}
$$

(3): Update $n \leftarrow n-\hat{N}_{j}, k \leftarrow k-K_{j}, j \leftarrow j-1$.

(4): If $j>1$ and $k \geq 0$, goto (2).

(5): For $m=1$ to $j$, set $\hat{N}_{m} \leftarrow\left\lfloor\frac{n}{j}\right\rfloor$.

(6): $\hat{N}_{j} \leftarrow \hat{N}_{j}+\operatorname{Rem}(n, j)$ where $\operatorname{Rem}(a, b)$ denotes the remainder of dividing $a$ by $b$.

The following lemma guarantees that the output of the above algorithm converges to the asymptotically optimal rate allocation introduced in Lemma III of section III-B.

Lemma IV. Consider a point-to-point connection over the Internet with $L$ independent paths from the source to the destination, each modeled as a Gilbert-Elliot cell. The paths are from $J$ different types, $L_{j}$ paths from the type $j$. Assume a 
block FEC $(N, K)$ is sent during an interval time $T$. For fixed values of $\gamma_{j}=\frac{L_{j}}{L}, n_{0}=\frac{N}{L}, k_{0}=\frac{K}{L}, T$ and asymptotically large number of paths $(L)$ we have

1) $\hat{P}_{e}(N, K, J) \doteq e^{-L \sum_{j=1} \gamma_{j} u_{j}(\alpha)}$

2) $\frac{\hat{N}_{j}}{N}= \begin{cases}0 & \text { if } \alpha \leq \mathbb{E}\left\{x_{j}\right\} \\ \frac{\gamma_{j} l_{j}(\alpha)}{\sum_{i=1, \alpha \leq \mathbb{E}\left\{x_{i}\right\}}^{J} \gamma_{i} l_{i}(\alpha)} & \text { otherwise }\end{cases}$

3) $K_{j}=\alpha \hat{N}_{j}$ for $\alpha>\mathbb{E}\left\{x_{j}\right\}$.

where $\alpha=\frac{k_{0}}{n_{0}}$, and $u_{j}()$ and $l_{j}()$ are defined in subsections III-A and III-B. The proof can be found in [13].

The proposed algorithm is compared with four other allocation schemes over $L=6$ paths in Fig. 2. The optimal method uses exhaustive search over all possible allocations. 'Best Path Allocation' assigns everything to the best path only, ignoring the rest. 'Equal Distribution' scheme distributes the packets among all paths equally. Finally, the 'Asymptotically Optimal' allocation assigns the rates based on the equation (8). The block length and the number of information packets are assumed to be $N=100$ and $K=90$, respectively. The overall rate is $S_{r e q}=1000 \mathrm{pkt} / \mathrm{sec}$, and the average good state duration is $\frac{1}{\mu_{g}}=1 \mathrm{~s}$ for all paths. The average duration of bad bursts for the six paths is listed as $\left[17.5 \mathrm{~ms} \pm \frac{\Delta}{2}, 17.5 \mathrm{~ms} \pm\right.$ $\left.\frac{3 \Delta}{2}, 17.5 \mathrm{~ms} \pm \frac{5 \Delta}{2}\right]$, such that the median is fixed at $17.5 \mathrm{~ms}$. $\Delta$ is also defined as a measure of deviation from this median.

As seen in a wide range, our suboptimal algorithm tracks the optimal algorithm so closely that their graphs are not easily distinguishable. However, the 'Asymptotically Optimal' rate allocation results in lower performance since there is only one path from each type which makes the asymptotic analysis assumptions invalid. When $\Delta=0$, 'Equal Distribution' scheme obviously coincides the optimal allocation. This scheme eventually diverges from the optimal algorithm as $\Delta$ grows. However, it still outperforms the best path allocation method as long as $\Delta$ is not too large. For very large values of $\Delta$, the best path dominates all the other ones, and the best path allocation eventually converges to the optimal scheme.

\section{CONClusion}

In this work, we study the performance of forward error correction over a block of packets sent through multiple independent paths. First, it is shown that MDS block codes are optimum over our Internet Channel model, and any other erasure channel. Then, it is shown that the probability of irrecoverable loss, $P_{E}$, decays exponentially with $L$. Furthermore, the optimal rate allocation problem is solved in the asymptotic case where $L$ is very large. It is seen that in the optimal rate allocation, each path is assigned a positive rate iff its quality is above a certain threshold. Finally, we focus on the problem of optimum path rate allocation when $L$ is not

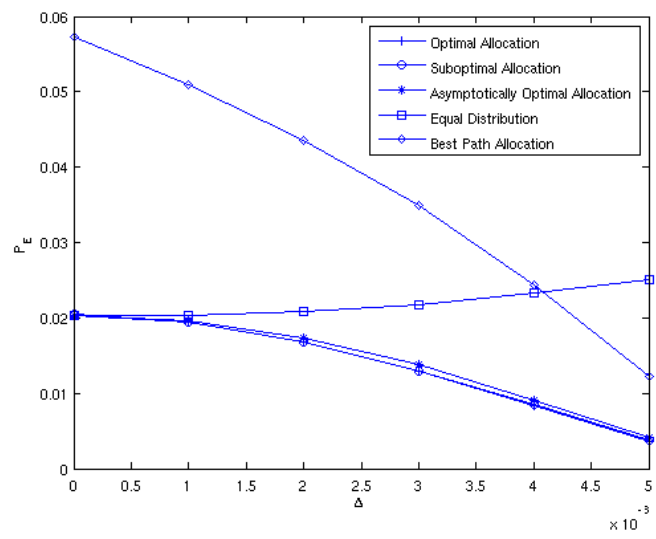

Fig. 2. Optimal and suboptimal rate allocations are compared with equal distribution and best path allocation schemes for different values of $\Delta$

necessarily large. A heuristic suboptimal algorithm is proposed which estimates the optimal allocation in polynomial time. For large values of $L$, the result of this algorithm converges to the optimal solution of the asymptotic analysis. Finally, the simulation results show that the proposed suboptimal algorithm approximates the optimal allocation very closely.

\section{REFERENCES}

[1] J.C. Bolot, S. Fosse-Parisis, and D. Towsley, "Adaptive FEC-based error control for Internet telephony," in IEEE INFOCOM, Proc. IEEE Vol. 3, 1999, pp. 1453-1460.

[2] T. Nguyen and A. Zakhor, "Multiple Sender Distributed Video Streaming," IEEE transactions on multimedia, vol. 6, no. 2, pp. 315-326, 2004.

[3] S. Mao, S.S. Panwar, and Y.T. Hou, "On optimal partitioning of realtime traffic over multiple paths," in INFOCOM 2005, Proc. IEEE Vol. 4, 2005, pp. 2325-2336.

[4] J. Han, D. Watson, and F. Jahanian, "An Experimental Study of Internet Path Diversity," IEEE Transactions on Dependable and Secure Computing, vol. 3, no. 4, pp. 273 - 288, 2006.

[5] Richard G. Ogier, Vlad Rutenburg, and Nauchum Shacham, "Distributed Algorithms for Computing Shortest Pairs of Disjoint Paths," IEEE transactions on information theory, vol. 39, no. 2, pp. 443-455, 1993.

[6] David G. Andersen, Resilient Overlay Networks. Master's Thesis, Massachusetts Institute of Technology, 2001.

[7] Roger Karrer, and Thomas Gross, "Multipath Streaming in Best-Effort Networks," in Proc. of the IEEE International Conference on Communications (ICC'03), 2003.

[8] J.G. Apostolopoulos, T. Wong, W. Tan, and S.J. Wee, "On Multiple Description Streaming with Content Delivery Networks," in IEEE INFOCOM, Proc. IEEE Vol. 3, 2002, pp. 1736 - 1745.

[9] Ron M. Roth, Introduction to Coding Theory, 1st ed. Cambridge University Press, 2006.

[10] W. T. Tan and A. Zakhor, "Video Multicast Using Layered FEC and Scalable Compression," IEEE Transactions on Circuits and Systems for Video Technology, vol. 11, no. 3, pp. 373-386, 2001.

[11] L. Dairaine, L. Lancrica, J. Lacan, and J. Fimes, "Content-Access QoS in Peer-to-Peer Networks Using a Fast MDS Erasure Code," Elsevier Computer Communications, vol. 28, no. 15, pp. 1778-1790, 2005.

[12] J. Padhye, V. Firoiu, D.F Towsley, and J.F. Kurose, "Modeling TCP Reno performance: a simple model and its empirical validation," IEEE/ACM Transactions on Networking, vol. 8, no. 2, pp. 133 - 145, 2000.

[13] S. Fashandi, S. Oveisgharan, and A.K. Khandani, "Path Diversity over the Internet: Performance Analysis and Rate Allocation," 2007, library and Archives Canada Technical Report UW-ECE \#2007-16, http://shannon2.uwaterloo.ca/r/2007-16_Fashandi.pdf.

[14] Amir Dembo and Ofer Zeitouni, Large Deviations Techniques and Applications, 2nd ed. New York: Springer, 1998. 